ARTÍCULO DE INVESTIGACIÓN

\title{
Síndrome metabólico, lipoproteína(a) y aterosclerosis subclínica en población mexicana
}

\author{
Metabolic syndrome, lipoprotein(a) and subclinical atherosclerosis in Mexican population \\ Guillermo C. Cardoso-Saldaña ${ }^{*}$, María del C. González-Salazar ${ }^{1}$, Rosalinda Posadas-Sánchez ${ }^{1}$ y \\ Gilberto Vargas-Alarcón ${ }^{2}$ \\ ${ }^{1}$ Departamento de Endocrinología; ${ }^{2}$ Departamento de Biología Molecular. Instituto Nacional de Cardiología Ignacio Chávez, Ciudad de México, \\ México
}

\section{Resumen}

Objetivo: Investigar la asociación del síndrome metabólico y la lipoproteína(a) [Lp(a)] con el riesgo de aterosclerosis subclínica en adultos mexicanos. Método: En 953 mujeres y hombres se evaluaron datos clínicos, bioquímicos y tomográficos de grasa abdominal visceral, subcutánea, hepática y calcio arterial coronario. La $L p(a)$ se determinó mediante nefelometría y el síndrome metabólico se diagnosticó con los criterios del Adult Treatment Panel III. La asociación independiente de estas variables con el calcio arterial coronario se obtuvo con análisis de regresión logística multivariada. Resultados: La edad, el peso, el índice de masa corporal, la presión arterial sistólica y diastólica, los volúmenes de grasa abdominal, los lípidos, la glucosa, la insulina y el índice de resistencia a insulina fueron significativamente mayores en los sujetos con síndrome metabólico, mientras que la mediana de $L p(a)$ fue más baja en comparación con los sujetos sin el síndrome (3.7 [rango intercuartílico (RIC): 2.3-9.2 vs. 5.9 [RIC: $2.5-13.1) \mathrm{mg} / \mathrm{dl} ; \mathrm{p}<0.01)$. El número de componentes y el síndrome metabólico se asociaron inversamente con la $\mathrm{Lp}(\mathrm{a})$ elevada (> $30 \mathrm{mg} / \mathrm{dl}$ ). La presencia de síndrome metabólico se asoció con un riesgo de calcio arterial coronario > 0 (odds ratio [OR]: 2.19; intervalo de confianza del 95\% [IC95\%]: 1.64-2.94; $p<0.001$ ), independientemente de la Lp(a) elevada. La glucemia > $100 \mathrm{mg} / \mathrm{dl}$ (OR: 2.42; IC95\%: 1.7-3.4; $p<0.0001)$ y la presión arterial elevada (OR: 2.14; IC95\%: 1.5-3.1; $p>0.0001$ ) se asociaron con calcio arterial coronario > 0. Conclusiones: En población mexicana existe una asociación inversa entre la concentración de $L p(a)$ y el síndrome metabólico. Este y sus componentes se asociaron positivamente con aterosclerosis subclínica. La elevada prevalencia de obesidad, diabetes, hipertensión arterial, triglicéridos elevados y concentración de colesterol unido a lipoproteínas de alta densidad que caracterizan a la población mexicana pudieran explicar las diferencias con otras poblaciones.

Palabras clave: Aterosclerosis subclínica. Lipoproteína (a). Síndrome metabólico.

\section{Abstract}

Objective: To assess the relationship of metabolic syndrome (MetS) and Lp(a) with subclinical atherosclerosis (CAC) in Mexican adults. Method: Clinical, biochemical and tomographic data of visceral, subcutaneous, hepatic abdominal fat and $C A C$ were evaluated in 953 women and men. $L p(a)$ was determined by nephelometry and MetS was diagnosed according to ATP III criteria. Multivariate logistic regression analysis was performed to determine the independent association of these

\section{Correspondencia:}

*Guillermo C. Cardoso-Saldaña E-mail: gccardosos@yahoo.com
Disponible en internet: $30-11-2020$ Arch Cardiol Mex. 2021;91(3):307-314 www.archivoscardiologia.com 1405-9940 / @ 2020 Instituto Nacional de Cardiología Ignacio Chávez. Publicado por Permanyer. Este es un artículo open access bajo la licencia CC BY-NC-ND (http://creativecommons.org/licenses/by-nc-nd/4.0/). 
variables with CAC. Results: Age, weight, body mass index, systolic and diastolic blood pressure, volumes of visceral, subcutaneous and hepatic abdominal fat, lipids, glucose, insulin and HOMA-RI were significantly higher in subjects with MetS. The median $L p(a)$ was lower in subjects with MetS compared to subjects without MetS (3.7 [IR: 2.3-9.2 vs. 5.9 [IR: 2.5-13.1) mg/dL; $p<0.01)$. The number of components and the MetS were inversely associated with the elevated $L p(a)(>30 \mathrm{mg} / \mathrm{dL})$. The presence of MetS was associated with a CAC risk >0 (OR: 2.19, [95\% Cl (1.64-2.94)]; $p<0.001)$, independently of elevated $L p(a)$. The components of MetS that were independently associated with the presence of CAC >0 UA were glycaemia $>100 \mathrm{mg} / \mathrm{dL}$ (OR 2.42, [95\% Cl (1.7-3.4)]; $p<0.0001)$ and high blood pressure (OR 2.14 [95\% Cl (1.5-3.1)]; $p<0.0001)$. Conclusions: In Mexican population there is an inverse association between $L p(a)$ levels and MetS. The MetS and its components were associated with subclinical atherosclerosis. The high prevalence of obesity, diabetes, high blood pressure high triglycerides and low HDL-C, characteristics of Mexican population could explain the differences with other populations.

Key words: Subclinical atherosclerosis. Lipoprotein (a). Metabolic syndrome.

\section{Introducción}

El síndrome metabólico (SMet) se caracteriza por resistencia a la insulina, exceso de peso, intolerancia a la glucosa, dislipidemia aterogénica (triglicéridos elevados y colesterol unido a lipoproteínas de alta densidad [C-HDL] bajo), inflamación y estado protrombótico ${ }^{1,2}$. Los estudios clínicos retrospectivos y los metaanálisis han mostrado que los pacientes con SMet tienen mayor riesgo de enfermedad arterial coronaria $(E A C)^{3,4}$. La lipoproteína(a) [Lp(a)] es un complejo macromolecular formado por una lipoproteína de baja densidad y la Apo(a), una proteína similar al plasminógeno ${ }^{5}$ que se une a la fibrina bloqueando la fibrinólisis y aumentando la trombogénesis ${ }^{6,7}$. Varios estudios han demostrado que la concentración elevada de $L p(a)$ es un factor de riesgo independiente de $E A C^{8-13}$. Sin embargo, la asociación con el depósito de calcio en las arterias coronarias es controversial: algunas evidencias son positivas ${ }^{14,15}$ y otras son negativas ${ }^{16,17}$. La inconsistencia de los resultados pudiera deberse principalmente a: 1) la amplia variación en la concentración de $L p(a)$ determinada genéticamente en los grupos étnicos; 2) las prevalencias de los polimorfismos del gen LPA asociados a concentraciones elevadas de $L p(a)^{18-20} ;$ y 3) las diferencias en la prevalencia de obesidad, diabetes, hipertensión y tabaquismo en los grupos estudiados. De acuerdo con la Encuesta Nacional de Salud y Nutrición 2018 (https://ensanut.insp. $\mathrm{mx} /$ encuestas/ ensanut2018/ informes.php) en población adulta mexicana, la prevalencia de SMet es del $45 \%$, la de exceso de peso (sobrepeso y obesidad) es del $75.2 \%$, la de diabetes mellitus es del $10.3 \%$, la de hipertensión es del $18.4 \%$ y la de tabaquismo es del $7.1 \%$. En un estudio previo en población mexicana, la prevalencia de $L p(a)$ elevada $\geq 30 \mathrm{mg} / \mathrm{dl}$ fue del $14 \%$, y en el $33 \%$ de los pacientes con EAC se asoció con infarto agudo al miocardio ${ }^{21}$. En un estudio en mestizos mexicanos sin antecedentes personales $\mathrm{ni}$ familiares de EAC, la prevalencia de calcificación arterial coronaria $(C A C)>0$, que es un factor de riesgo independiente de $\mathrm{EAC}^{22}$, fue del $27 \%{ }^{23}$.

Considerando que las prevalencias de SMet y L(a) $\geq 30 \mathrm{mg} / \mathrm{dl}$ son elevadas en nuestra población, y que el efecto de la coexistencia de ambos factores de riesgo sobre la aterosclerosis subclínica se desconoce, el propósito de este estudio fue investigar en sujetos sin antecedentes personales ni familiares de EAC la asociación del SMet y la concentración de $L p(a)$ con la presencia de aterosclerosis subclínica evaluada por el depósito de CAC.

\section{Método}

\section{Sujetos estudiados}

Se incluyeron 953 sujetos del proyecto Genética de la Enfermedad Aterosclerosa (GEA) ${ }^{24}$, diseñado en el Instituto Nacional de Cardiología Ignacio Chávez para investigar la asociación de factores genéticos con factores de riesgo cardiovascular tradicionales y emergentes en población mexicana adulta de la Ciudad de México sin antecedentes de EAC prematura, con una edad entre 35 y 70 años, en sujetos reclutados de los donadores que acuden al banco de sangre de dicho Instituto o mediante invitación en centros de salud del área metropolitana de la Ciudad de México. Ninguno de los participantes presentó enfermedad hepática, renal, tiroidea u oncológica. El protocolo de estudio fue aprobado por el Comité de Ética del Instituto Nacional de Cardiología y diseñado de acuerdo con las guías de la Declaración de Helsinki. Todos los participantes firmaron el consentimiento informado.

A todos los sujetos se les aplicaron cuestionarios validados para obtener información demográfica, antecedentes personales y familiares de factores de riesgo 
para EAC, actividad física y uso de medicamentos. El índice de masa corporal (IMC) se obtuvo con la fórmula: peso $(\mathrm{kg})$ / estatura $\left(\mathrm{m}^{2}\right)$. El perímetro de la cintura (cm) se midió con una cinta métrica de fibra de vidrio, colocada en el punto medio entre la última costilla y la cresta ilíaca, con una aproximación de $0.5 \mathrm{~cm}$.

La frecuencia cardiaca y la presión arterial sistólica y diastólica se midieron en tres ocasiones con intervalos de 1 minuto, después de 10 minutos de reposo en posición sedente. Para los análisis estadísticos se usó el promedio de la segunda y la tercera mediciones. La hipertensión arterial se definió de acuerdo con las guías del Seventh Report of the Joint National Committee on Prevention, Detection, Evaluation and Treatment of High Blood Pressure ${ }^{25}$. Se consideraron fumadores activos los sujetos que fumaban al menos un cigarro al día o siete a la semana durante los 6 meses previos al estudio. La actividad física durante el trabajo y el tiempo libre se evaluó usando un cuestionario validado internacionalmente ${ }^{26}$.

Las muestras de sangre de los participantes se obtuvieron por la mañana con un ayuno mínimo de 10 horas, sin haber practicado ejercicio antes de la punción venosa y después de 20 minutos en reposo en posición sedente. Los valores de glucosa, colesterol total (CT), triglicéridos (TG), C-HDL, Apo Al y B100 se determinaron en plasma con métodos enzimáticos colorimétricos (Roche/Hitachi, Germany) en un autoanalizador Hitachi 902 (Hitachi LTD, Tokyo Japan). El colesterol unido a lipoproteínas de baja densidad (C-LDL) se calculó con la fórmula de Friedewald modificada por DeLong, et al. ${ }^{27}$, y el colesterol no HDL se calculó con la diferencia entre el CT menos el C-HDL (colesterol no $\mathrm{HDL}=\mathrm{CT}-\mathrm{C}-\mathrm{HDL}$ ).

La concentración de insulina se determinó por radioinmunoanálisis (Millipore, St. Charles, Missouri, USA) y la resistencia a la insulina se estimó con el modelo homeostático HOMA-RI (HOMA-RI = insulina $[\mathrm{uUl} / \mathrm{ml}] \times$ glucosa [mmol] / 22.5 $)^{28}$.

La presencia de dislipidemia se definió, de acuerdo con los criterios del National Cholesterol Education Program Adult Treatment Panel III (NCEP-ATP III) ${ }^{1}$, como hipercolesterolemia (CT $\geq 200$ o C-LDL $\geq 160$ $\mathrm{mg} / \mathrm{dl}$ ), hipertrigliceridemia (TG $\geq 150 \mathrm{mg} / \mathrm{dl}$ ) y hipoalfalipoproteinemia (C-HDL $<40 \mathrm{mg} / \mathrm{dl}$ en hombres $\mathrm{y}<$ $50 \mathrm{mg} / \mathrm{dl}$ en mujeres). La precisión y la exactitud en las mediciones de lípidos y lipoproteínas fueron certificadas periódicamente con el programa Lipid Standardization Program of the Center for Disease Control and Prevention (LSP-CDC, Atlanta, GA, USA). LoS coeficientes de variación intraanálisis e interanálisis fueron $<3 \%$.

La concentración de $L p(a)$ se determinó mediante inmunonefelometría cinética con el reactivo $\mathrm{N}$ Latex Lp(a) (Siemens Healthcare Diagnostics Products $\mathrm{GmbH}$, Marburg, Germany) ${ }^{29}$ en un nefelómetro automatizado BN ProSpec® con calibradores, controles y reactivos de la misma marca. Los coeficientes de variación intraanálisis e interanálisis fueron $<6 \%$.

El SMet se definió de acuerdo con las guías NCEPATP III si el participante presentaba al menos tres de los cinco componentes: 1) circunferencia de cintura $\geq 90 \mathrm{~cm}$ en hombres $0 \geq 80 \mathrm{~cm}$ en mujeres; 2) TG $\geq 150 \mathrm{mg} / \mathrm{dl}$; 3) C-HDL $<40 \mathrm{mg} / \mathrm{dl}$ en hombres y $<50 \mathrm{mg} / \mathrm{dl}$ en mujeres; 4) presión arterial sistólica $\geq$ $130 \mathrm{mmHg}$ y diastólica $\geq 85 \mathrm{mmHg}$, o tratamiento farmacológico antihipertensivo; y 5) glucosa plasmática en ayuno $\geq 126 \mathrm{mg} / \mathrm{dll}$, o diagnóstico o tratamiento previo de diabetes mellitus.

\section{Determinaciones tomográficas}

La tomografía computada es un método validado para la medición del tejido adiposo visceral y la $\mathrm{CAC}^{30}$. En este estudio, la tomografía de tórax y abdomen se realizó utilizando un sistema helicoidal multidetector de 64 canales (Somatom Cardiac Sensation 64, Forchheim, Bavaria, Germany) y la interpretaron radiólogos experimentados. Las imágenes del tomógrafo fueron evaluadas para cuantificar el tejido adiposo abdominal total, subcutáneo y visceral|31,32. El depósito de CAC se expresó en unidades Agatston (UA ${ }^{33}$. Se consideraron todas las áreas con atenuación > 130 unidades Hounsfield, y con la sumatoria de las puntuaciones individuales de las arterias coronarias se obtuvo la puntuación total de Agatston. El coeficiente de correlación intraobservador fue de $0.99(p<0.001)$.

\section{Análisis estadístico}

Los datos se expresan como el promedio y la desviación estándar o como la mediana con el rango intercuartílico (RIC) para las variables con distribución asimétrica, y como porcentajes las variables categóricas. La comparación de variables continuas se realizó con la prueba $t$ de Student 0 con la prueba $U$ de Mann-Whitney, según el tipo de distribución. Las variables categóricas se compararon con la prueba de ji al cuadrado.

El depósito de calcio coronario se analizó como variable categórica (presencia o ausencia de CAC 
Tabla 1. Características de los sujetos según la presencia o no de síndrome metabólico

\begin{tabular}{|c|c|c|c|}
\hline & Sin SMet (n = 555) & Con SMet $(n=398)$ & p \\
\hline Edad, años* & $52.7 \pm 9.6$ & $54.1 \pm 8.8$ & 0.006 \\
\hline Hombres $(\%)$ & 52.1 & 47.9 & $<0.001$ \\
\hline $\mathrm{IMC}, \mathrm{kg} / \mathrm{m}^{2^{*}}$ & $27.3 \pm 4.1$ & $29.9 \pm 4.1$ & $<0.001$ \\
\hline Cintura, $\mathrm{cm}^{*}$ & $90.7 \pm 11.3$ & $98.7 \pm 10.4$ & $<0.001$ \\
\hline $\mathrm{GAV}, \mathrm{mm}^{3 *}$ & $138.2 \pm 60.8$ & $182.3 \pm 61.1$ & $<0.001$ \\
\hline $\mathrm{GAS}, \mathrm{mm}^{3 *}$ & $112.3 \pm 14.4$ & $123.7 \pm 19.0$ & $<0.001$ \\
\hline PAS, $\mathrm{mmHg}^{*}$ & $112.3 \pm 14.4$ & $123.7 \pm 19.0$ & $<0.001$ \\
\hline $\mathrm{PAD}, \mathrm{mmHg}{ }^{*}$ & $69.8 \pm 8.3$ & $75.4 \pm 9.8$ & $<0.001$ \\
\hline $\mathrm{CT}, \mathrm{mg} / \mathrm{dl}{ }^{*}$ & $188.8 \pm 32.9$ & $199.2 \pm 40.0$ & $<0.001$ \\
\hline C-LDL, mg/dl* & 115.0. \pm 28.3 & $123.7 \pm 35.1$ & 0.003 \\
\hline $\mathrm{TG}, \mathrm{mg} / \mathrm{dl}^{*}$ & $130.9 \pm 58.8$ & $236.2 \pm 193.7$ & $<0.001$ \\
\hline C-HDL, mg/dl* & $51.5 \pm 13.0$ & $38.5 \pm 9.6$ & $<0.001$ \\
\hline Glucosa, mg/dl ${ }^{\dagger}$ & $88(82-93)$ & $96.5(88-109.2)$ & $<0.001$ \\
\hline Insulina, $\mu \mathrm{IU} / \mathrm{ml}^{\dagger}$ & $14.4(10.6-19.9)$ & $20.5(15.6-27.1)$ & $<0.001$ \\
\hline $\mathrm{HOMA}^{-\mathrm{RI}^{\dagger}}$ & $3.1(2.2-4.4)$ & $5.3(3.8-7.6)$ & $<0.001$ \\
\hline $\mathrm{Lp}(\mathrm{a}), \mathrm{mg} / \mathrm{dl}^{\dagger}$ & $5.9(2.5-13.1)$ & $3.7(2.3-9.2)$ & $<0.001$ \\
\hline $\mathrm{CAC}, \mathrm{UA}^{\dagger}$ & $0.0(0.0-0.0)$ & $0.0(0.0-11.3)$ & $<0.001$ \\
\hline Lp(a) $\geq 30$ mg/dl (\%) & 10.8 & 7.5 & 0.087 \\
\hline CAC >0 UA $(\%)$ & 23.6 & 39.9 & $<0.001$ \\
\hline Riesgo ASCVD (\% a10 años) ${ }^{\dagger}$ & $1.2(0.8-2.0)$ & $1.6(1-3.2)$ & $<0.001$ \\
\hline
\end{tabular}

ASCVD: Enfermedad arterial coronaria; CAC: calcio arterial coronario; C-HDL: colesterol en lipoproteínas de alta densidad; C-LDL: colesterol en lipoproteínas de baja densidad; CT: colesterol total; GAV: grasa abdominal visceral; GAS: grasa abdominal subcutánea; HOMA-RI: resistencia a la insulina; IMC: índice de masa muscular; PAD: presión arterial diastólica; PAS: presión arterial sistólica; SMet: síndrome metabólico; TG: triglicéridos.

*Promedio \pm desviación estándar.

†Mediana (rango intercuartílico).

$>0$ UA). Para investigar la independencia de las asociaciones entre la $\mathrm{Lp}(\mathrm{a}) \geq 30 \mathrm{mg} / \mathrm{dl}$, el SMet y la presencia de CAC > 0 UA se realizó un análisis de regresión múltiple ajustado por sexo y edad, y luego ajustado por IMC, TG, C-LDL, diabetes mellitus, hipertensión y tabaquismo. Un valor de $p<0.05$ se consideró estadísticamente significativo. Todos los análisis estadísticos se realizaron con SPSS V.16.

\section{Resultados}

Las características de los 953 sujetos sin y con SMet se muestran en la tabla 1 . El $41.7 \%(n=398)$ de los participantes fueron diagnosticados de SMet. En los sujetos con SMet, el promedio o la mediana de las variables antropométricas, como el IMC, el perímetro de la cintura, el volumen de grasa abdominal visceral y subcutánea, y de las variables bioquímicas, como las concentraciones de lípidos, lipoproteínas, glucosa e insulina, y el índice de resistencia a la insulina (HOMA-RI), fueron significativamente mayores que en aquellos sin SMet, mientras que el promedio del C-HDL fue significativamente menor $(p<0.001)$ (Tabla 1).

La mediana de $L p(a)$ fue significativamente más baja en los sujetos con SMet: 3.7 (RIC: 2.3-9.2) vs. 5.9 (RIC: $2.5-13.1) ; p<0.001$. Comparados con los sujetos sin SMet, la prevalencia de $L p(a) \geq 30 \mathrm{mg} / \mathrm{dl}$ fue más baja en los sujetos con SMet (7.5 vs. $10.8 \% ; p=0.087$ ). Sin embargo, la prevalencia de aterosclerosis subclínica (CAC > 0 UA) en los sujetos sin SMet fue significativamente menor que la de los sujetos con SMet (23.6 vs. $39.9 \%$; $p<0.001$ ) (Tabla 1 ).

Como se muestra en la figura 1 , la prevalencia de $L p(a) \geq 30 \mathrm{mg} / \mathrm{dl}$ se asocia de manera inversa, y la 


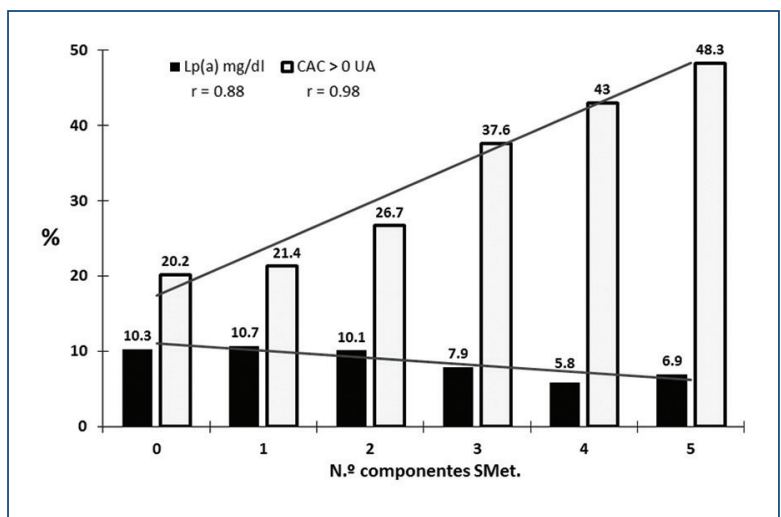

Figura 1. Prevalencia de lipoproteína(a) $[\mathrm{Lp}(\mathrm{a})] \geq 30 \mathrm{mg} / \mathrm{dl}$ y de calcio arterial coronario (CAC) > 0 UA en población mexicana según el número de componentes del síndrome metabólico (SMet).

prevalencia de CAC de manera positiva, con el número de componentes del SMet. El porcentaje de sujetos con $\mathrm{Lp}(\mathrm{a})$ elevada disminuyó y el de CAC >0 UA se incrementó, progresivamente, con la presencia de uno a cinco de los componentes del SMet. Estas relaciones fueron evaluadas con análisis de regresión logística para investigar la independencia de la asociación entre los componentes del SMet y la concentración de Lp(a) $\geq 30 \mathrm{mg} / \mathrm{dl}$ y la presencia de CAC >0 UA. En los sujetos con SMet, con una glucemia $\geq 100 \mathrm{mg} / \mathrm{dl}$ o una presión arterial $\geq 130 / 85 \mathrm{mmHg}$, el riesgo de CAC > 0 UA fue dos veces mayor que en los sujetos sin SMet (odds ratio [OR]: 2.19, intervalo de confianza del 95\% [IC95\%]: 1.6-2.6, $p<0.001$; OR: 2.4, IC95\%: 1.7-3.4, p < 0.001; y OR: 2.1, IC95\%: 1.6-3.1, p < 0.001; respectivamente). Los TG $\geq 150 \mathrm{mg} / \mathrm{dl}$, la concentración baja de $\mathrm{C}$-HDL $(<50 \mathrm{mg} / \mathrm{dl}$ en mujeres $\mathrm{y}<40 \mathrm{mg} / \mathrm{dl}$ en hombres) y la obesidad (perímetro de cintura) no se asociaron con mayor riesgo de CAC >0 UA (Fig. 2).

\section{Discusión}

La $L p(a)$ es un factor de riesgo independiente para $\mathrm{EAC}^{34}$; no obstante, el grado de asociación con la aterosclerosis varía ampliamente entre los diferentes grupos étnicos. En este estudio transversal en 953 mexicanos sin antecedentes personales ni familiares de EAC prematura, la mediana y la prevalencia de $L p(a) \geq 30 \mathrm{mg} / \mathrm{dl}$ fueron significativamente menores en los sujetos con SMet, mientras que la prevalencia de CAC > 0 UA fue significativamente mayor. La prevalencia de CAC $>0 \cup A$ se incrementó, progresivamente, con la presencia de uno

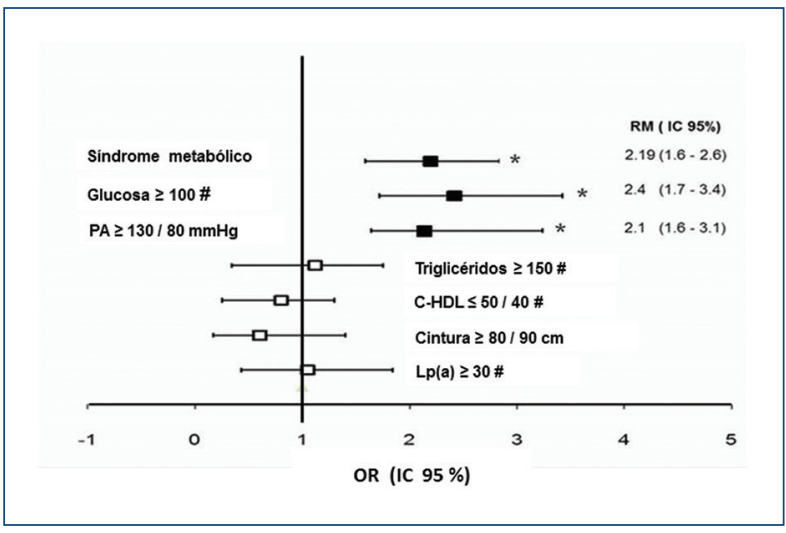

Figura 2. Riesgo de aterosclerosis subclínica (calcio arterial coronario $>0 \mathrm{UA}$ ) en sujetos con síndrome metabólico y sus componentes en población mexicana. Análisis de regresión múltiple ajustado por sexo, edad, colesterol en lipoproteínas de baja densidad, tabaquismo y actividad física. C-HDL: colesterol en lipoproteínas de alta densidad; IC95\%: intervalo de confianza del 95\%; OR: odds ratio; PA: presión arterial.

a cinco de los componentes del SMet. Sin embargo, el porcentaje de sujetos con Lp(a) elevada disminuyó y no se asoció con CAC. Las medidas de adiposidad corporal, como el IMC y la grasa abdominal visceral y subcutánea, y los marcadores cardiometabólicos, como la insulina en plasma y HOMA-RI, fueron significativamente mayores en los sujetos con SMet, pero la glucemia $>100 \mathrm{mg} / \mathrm{dl}$ y la hipertensión arterial $>$ 130/85 mmHg fueron los únicos componentes del SMet que se asociaron, de manera independiente y significativa, con la presencia de aterosclerosis subclínica.

El consenso ATP III del año $2014^{35}$ sugiere que la primera diana terapéutica para el tratamiento de la aterosclerosis es la concentración elevada de C-LDL, y la siguiente debe ser el SMet, debido a que cada uno de sus componentes es un factor de riesgo de EAC. La concentración elevada de $L p(a)$ es un factor de riesgo emergente para $\mathrm{EAC}^{13,36,37}$. Se ha sugerido que la coexistencia de la Lp(a) elevada con el SMet pudiera potenciar el riesgo de aterosclerosis prematura o acelerar el proceso ateroscleroso ${ }^{38}$; sin embargo, los estudios en los que se ha investigado esta asociación son escasos y los resultados son controversiales. En la gran mayoría de los estudios se analizan de manera aislada cada uno de los componentes del SMet y su asociación con la concentración de Lp(a). En pacientes cardiópatas con y sin SMet se encontró que los que presentaban SMet tenían concentraciones de Lp(a) 1.8 veces mayores que aquellos sin SMet (mediana de 
29.2 vs. $16.2 \mathrm{mg} / \mathrm{dl} ; \mathrm{p}<0.0001)$ y una prevalencia 2.3 veces mayor de $L p(a) \geq 30 \mathrm{mg} / \mathrm{dl}$ (51.4 vs. $21.6 \%$; $p<0.001)^{39}$. En otro estudio se encontró que las concentraciones medias de $\mathrm{Lp}(\mathrm{a})(5-17 \mathrm{mg} / \mathrm{dl})$ en mujeres, comparadas con los hombres con SMet, se asocian con progresión de la aterosclerosis ${ }^{38}$. Sin embargo, se ha informado que las concentraciones de $L p(a)$ son significativamente más bajas en los pacientes con SMet comparados con los sujetos sin SMet, y que la $L p(a)$ es un predictor de eventos cardiovasculares en sujetos $\sin \mathrm{SMet}^{40}$ o que en los sujetos con mayor concentración de Lp(a), el riesgo de SMet es significativamente menor ${ }^{41}$. Estos autores sugieren que la relación inversa entre el SMet y la $L p(a)$ pudiera deberse a un sesgo de sobrevida en los sujetos con antecedentes de EAC. La presencia de $L p(a) \geq 30 \mathrm{mg} / \mathrm{dl}$ y de SMet a edades tempranas pudiera aumentar la mortalidad en sujetos jóvenes, lo que explicaría la mayor proporción de Lp(a) baja entre los sobrevivientes con mayor edad $^{41}$.

Nuestros datos en sujetos sin EAC, agrupados por década de edad, mostraron que desde los 20 hasta los 70 años la prevalencia de $L p(a)$ elevada es mayor en los sujetos sin SMet, y por tanto no apoya la hipótesis del efecto de sobrevivencia que intenta explicar la relación inversa entre estos dos factores de riesgo de EAC; de hecho, la prevalencia de $L p(a) \geq 30 \mathrm{mg} / \mathrm{dl}$ no se asocia con el SMet ni con la aterosclerosis subclínica en ningún rango de edad. Resultados similares han sido informados por otros investigadores ${ }^{42}$.

En otros estudios en los que se incluyeron pacientes con $\mathrm{EAC}^{43} \mathrm{o}$ con dislipidemias ${ }^{44}$ se ha observado una asociación inversa entre las concentraciones de Lp(a) con TG elevados y con C-HDL bajo. Los autores sugieren que la asociación pudiera deberse a un mecanismo metabólico compartido entre los tres factores bioquímicos. En el presente estudio se observó una correlación negativa y significativa entre los TG y la $L p(a)(r=-0.073 ; p=0.024)$, que se perdió al agrupar la muestra en sujetos con y sin SMet. Estos resultados confirman lo reportado en otro estudio ${ }^{45}$ y apoyan los resultados, in vitro, en los que se demuestra que la Lp(a) es ligando del receptor de lipoproteínas de muy baja densidad en las membranas de células del endotelio, los macrófagos, las células espumosas y las células musculares lisas en las placas aterosclerosas. La internalización de la $L p(a)$ a estas células pudiera contribuir de manera importante al catabolismo de la $L p(a)$ y al desarrollo de las placas aterosclerosas ${ }^{46}$. Está bien documentado que la $L p(a)$ disminuye los efectos del PAI-1 y la formación de plasmina, generando un estado protrombótico. En los sujetos con SMet existen alteraciones en la hemostasia que disminuyen la fibrinólisis y favorecen la coagulación ${ }^{47}$. Se ha sugerido que el incremento de la concentración del PAl-1 en los sujetos con SMet pudiera disminuir la síntesis de $\operatorname{Lp}(a)^{48,49}$. El mecanismo fisiopatológico de la relación entre el SMet, la $L p(a)$ y la aterosclerosis se desconoce; sin embargo, un estudio prospectivo en sujetos con SMet y Lp(a) elevada pudiera generar información que permita conocer, al menos en parte, la relación entre estos factores de riesgo cardiovascular.

Este estudio presenta como limitaciones que es de tipo transversal, por lo que solo se pueden establecer asociaciones entre las variables y no es posible establecer la causalidad. El estudio incluyó voluntarios residentes en el área conurbada de la Ciudad de México y no es una muestra representativa de la población mexicana; sin embargo, la prevalencia de los factores de riesgo de EAC observados en este estudio es similar a la encontrada en la Encuesta Nacional de Salud y Nutrición 2016 (ENSANUT MC 2016), estudio aleatorizado y representativo de ámbito nacional ${ }^{50}$. Entre las fortalezas del estudio se encuentran el tamaño de muestra (mayor que en otros estudios), que los sujetos estudiados son de ambos sexos, con edades de $20 \mathrm{a}$ 70 años y sin antecedentes personales ni familiares de aterosclerosis, lo que disminuye el sesgo en la interpretación de los resultados, y la evaluación de la aterosclerosis subclínica (CAC) en el total de la muestra.

\section{Conclusiones}

En este estudio en población mexicana asintomática y sin antecedentes de enfermedad arterial coronaria se encontró: 1) una asociación inversa entre la concentración de Lp(a) con el SMet y sus componentes; 2) la concentración elevada de $L p(a)$ en sujetos con SMet no se asocia con aterosclerosis subclínica por el CAC; y 3) la elevada prevalencia de sobrepeso, obesidad, diabetes mellitus, presión arterial elevada y dislipidemia aterogénica (TG elevados y C-HDL bajo) que caracteriza la población mexicana pudiera explicar las diferencias con otras poblaciones.

\section{Agradecimientos}

Los autores quieren mostrar su agradecimiento a los sujetos del estudio, así como al personal que participó en el proyecto Genética de la Enfermedad Aterosclerótica (GEA). 


\section{Conflictos de intereses}

Ninguno.

\section{Financiamiento}

Este trabajo fue parcialmente apoyado por el Consejo Nacional de Ciencia y Tecnología (CONACyT Fronteras de la Ciencia No. 2016-01-1958).

\section{Responsabilidades éticas}

Protección de personas y animales. Los autores declaran que los procedimientos seguidos se conformaron a las normas éticas del comité de experimentación humana responsable y de acuerdo con la Asociación Médica Mundial y la Declaración de Helsinki.

Confidencialidad de los datos. Los autores declaran que han seguido los protocolos de su centro de trabajo sobre la publicación de datos de pacientes.

Derecho a la privacidad y consentimiento informado. Los autores han obtenido el consentimiento informado de los pacientes y/o sujetos referidos en el artículo. Este documento obra en poder del autor de correspondencia.

\section{Bibliografía}

1. Third Report of the National Cholesterol Education Program (NCEP) Expert Panel on Detection, Evaluation, and Treatment of High Blood Cholesterol in Adults (Adult Treatment Panel III) final report. Circulation. 2002;106:3143-21.

2. Saely $\mathrm{CH}$, Aczel S, Marte T, Langer P, Hoefle G, Drexel H, et al. The metabolic syndrome, insulin resistance, and cardiovascular risk in diabetic and nondiabetic patients. J Clin Endocrinol Metab. 2005;90:5698-703.

3. Galassi A, Reynolds K, He J. Metabolic syndrome and risk of cardiovascular disease: a meta-analysis. Am J Med. 2006;119:812-9.

4. Lu J, Wang L, Li M, Xu Y, Jiang Y, Wang W, et al. Metabolic syndrome among adults in China: The 2010 China Non Communicable Disease Surveillance. J Clin Endocrinol Metab. 2017;102:507-15.

5. McLean JW, Tomlinson JE, Kuang WJ, Eaton DL, Chen EY, Fless GM, et al. cDNA sequence of human apolipoprotein(a) is homologous to plasminogen. Nature. 1987;330:132-7.

6. Rouy D, Grailhe P, Nigon F, Chapman J, Anglés-Cano E. Lipoprotein(a) impairs generation of plasmin by fibrin-bound tissue-type plasminogen activator. In vitro studies in a plasma milieu. Arterioscler Thromb. 1991;11:629-38.

7. Hervio L, Chapman MJ, Thillet J, Loyau S, Anglés-Cano E. Does apolipoprotein(a) heterogeneity influence lipoprotein(a) effects on fibrinolysis? Blood. 1993;82:392-7

8. Clarke R, Peden JF, Hopewell JC, Kyriakou T, Goel A, Heath SC, et al. Genetic variants associated with $L p(a)$ lipoprotein level and coronary disease. N Engl J Med. 2009;361:2518-28.

9. Kamstrup PR, Tybjaerg-Hansen A, Steffensen R, Nordestgaard BG. Genetically elevated lipoprotein(a) and increased risk of myocardial infarction. JAMA. 2009;301:2331-9.

10. Tsimikas S, Hall JL. Lipoprotein(a) as a potential causal genetic risk factor of cardiovascular disease: a rationale for increased efforts to understand its pathophysiology and develop targeted therapies. J Am Coll Cardiol. 2012;60:716-21.

11. Nordestgaard BG, Chapman MJ, Ray K, Borén J, Andreotti F, Watts GF, et al. Lipoprotein(a) as a cardiovascular risk factor: current status. Eur Heart J. 2010;31:2844-53.

12. Dubé JB, Boffa MB, Hegele RA, Koschinsky ML. Lipoprotein(a): more interesting than ever after 50 years. Curr Opin Lipidol. 2012;23:133-40.

13. Danesh J, Collins R, Peto R. Lipoprotein(a) and coronary heart disease. Meta-analysis of prospective studies. Circulation. 2000;102:1082-5.
14. Superko HR, Hecht HS. Metabolic disorders contribute to subclinical coronary atherosclerosis in patients with coronary calcification. Am J Cardiol. 2001;88:260-4.

15. Verweij SL, de Ronde MWJ, Verbeek R, Boekholdt SM, Planken RN, Stroes ESG, et al. Elevated lipoprotein(a) levels are associated with coronary artery calcium scores in asymptomatic individuals with a family history of premature atherosclerotic cardiovascular disease. J Clin Lipidol. 2018:12:597-603.

16. Mahoney LT, Burns TL, Stanford W, Thompson BH, Witt JD, Rost CA, et al. Coronary risk factors measured in childhood and young adult life are associated with coronary artery calcification in young adults: the Muscatine Study. J Am Coll Cardiol. 1996;27:277-84

17. Nishino M, Malloy MJ, Naya-Vigne J, Russell J, Kane JP, Redberg RF. Lack of association of lipoprotein(a) levels with coronary calcium deposits in asymptomatic postmenopausal women. J Am Coll Cardiol. 2000;35: 314-20.

18. Sharrett AR, Ballantyne CM, Coady SA, Heiss G, Sorlie PD, Catellier D, et al. Coronary heart disease prediction from lipoprotein cholesterol levels, triglycerides, lipoprotein(a), apolipoproteins $A-I$ and $B$, and HDL density subfractions: The Atherosclerosis Risk in Communities (ARIC) Study. Circulation. 2001;104:110813.

19. Qasim AN, Martin SS, Mehta NN, Wolfe ML, Park J, Schwartz S, et al. Lipoprotein (a) is strongly associated with coronary artery calcification in type-2 diabetic women. Int J Cardiol. 2011;150:17-21.

20. Shai I, Schulze MB, Manson JE, Stampfer MJ, Rifai N, Hu FB. A prospective study of lipoprotein(a) and risk of coronary heart disease among women with type 2 diabetes. Diabetologia. 2005;48:1469-76.

21. Cardoso-Saldana G, Ize-Lema I, Kimura LY, Zamora González J, Posadas Romero C. Lipoprotein(a) and cardiovascular risk in adult Mexicans. Rev Invest Clin. 1997;49:85-92.

22. Budoff MJ, Shaw LJ, Liu ST, Weinstein SR, Mosler TP, Tseng PH, et al. Long-term prognosis associated with coronary calcification: observations from a registry of 25,253 patients. J Am Coll Cardiol. 2007;49:1860-70.

23. Posadas-Romero C López-Bautista F, Rodas-Díaz MA, Posadas-Sánchez R, Kimura-Hayama E, Juárez-Rojas JG, et al. Prevalence and extent of coronary artery calcification in an asymptomatic cardiovascular Mexican population: Genetics of Atherosclerotic Disease study. Arch Cardiol Mex. 2017;87:292-301.

24. Villarreal-Molina T, Posadas-Romero $C$, Romero-Hidalgo $S$, Antúnez-Argüelles E, Bautista-Grande A, Vargas-Alarcón G, et al. The ABCA1 gene $\mathrm{R} 230 \mathrm{C}$ variant is associated with decreased risk of premature coronary artery disease: the Genetics of Atherosclerotic Disease (GEA) Study. PLoS One. 2012;7:e49285

25. Chobanian AV, Bakris GL, Black HR, Cushman WC, Green LA Izzo JL Jr, et al. The Seventh Report of the Joint National Committee on Prevention, Detection, Evaluation, and Treatment of High Blood Pressure: the JNC 7 report. JAMA. 2003;289:2560-72.

26. Baecke JA, Burema J, Frijters JE. A short questionnaire for the measurement of habitual physical activity in epidemiological studies. Am J Clin Nutr. 1982;36:936-42.

27. DeLong DM, DeLong ER, Wood PD, Lippel K, Rifkind BM. A comparison of methods for the estimation of plasma low- and very low-density lipoprotein cholesterol. The Lipid Research Clinics Prevalence Study. JAMA. 1986;256:2372-7.

28. Matthews D, Hosker J, Rudenski A, Naylor BA, Treacher DF, Turner RC Homeostasis model assessment: insulin resistance and 0-cell function from fasting plasma glucose and insulin concentrations in man. Diabetologia. 1985;28:412-9.

29. Marcovina SM, Albers JJ, Scanu AM, Kennedy H, Giaculli F, Berg K, et al. Use of a reference material proposed by the International Federation of Clinical Chemistry and Laboratory Medicine to evaluate analytical methods for the determination of plasma lipoprotein(a). Clin Chem. 2000;46:1956-67.

30. Maurovich-Horvat P, Massaro J, Fox CS, Moselewski F, O'Donnell CJ, Hoffmann U. Comparison of anthropometric, area- and volume-based assessment of abdominal subcutaneous and visceral adipose tissue volumes using multi-detector computed tomography. Int $\mathrm{J}$ Obes. 2007;31:500-6.

31. Kvist H, Chowdhury B, Grangard U, Tylén U, Sjöström L. Total and visceral adipose-tissue volumes derived from measurements with computed tomography in adult men and women: predictive equations. Am J Clin Nutr. 1988;48:1351-61.

32. Armellini F, Zamboni M, Castelli S, Micciolo R, Mino A, Turcato E, et al. Measured and predicted total and visceral adipose tissue in women. Correlations with metabolic parameters. Int J Obes Relat Metab Disord. 1994:18:641-7.

33. Mautner GC, Mautner SL, Froehlich J, Feuerstein IM, Proschan MA, Roberts WC, et al. Coronary artery calcification: assessment with electron beam CT and histomorphometric correlation. Radiology. 1994;192:619-23.

34. Nordestgaard BG, Chapman MJ, Ray K, Borén J, Andreotti F, Watts GF, et al. Lipoprotein(a) as a cardiovascular risk factor: current status. Eur Heart J. 2010;31:2844-53. 
35. Eckel RH, Cornier M-A. Update on the NCEP ATP-III emerging cardiometabolic risk factors. BMC Med. 2014;12:115.

36. Craig WY, Neveux LM, Palomaki GE, Cleveland MM, Haddow JE. Lipoprotein(a) as a risk factor for ischemic heart disease: metaanalysis of prospective studies. Clin Chem. 1998;44:2301-6.

37. Luc G, Bard J-M, Arveiler D, Ferrieres J, Evans A, Amouyel P, et al. Lipoprotein (a) as a predictor of coronary heart disease: the PRIME Study. Atherosclerosis. 2002;163:377-84.

38. Onat A, Hergenc G, Ozhan H, Kaya Z, Bulur S, Ayhan E, et al. Lipoprotein(a) is associated with coronary heart disease independent of metabolic syndrome. Coron Artery Dis. 2008;19:125-31.

39. Bozbas H, Yildirir A, Pirat B, Eroğlu S, Korkmaz ME, Atar I, et al. Increased lipoprotein(a) in metabolic syndrome: is it a contributing factor to premature atherosclerosis? Anadolu Kardiyol Derg. 2008:8:111-5.

40. Vonbank A, Saely CH, Rein P, Zanolin D, Drexel H. Lipoprotein(a), the metabolic syndrome and vascular risk in angiographied coronary patients. $\mathrm{J}$ Clin Endocrinol Metab. 2016;101:3199-203.

41. Wu XY, Lin L, Qi HY, Du R, Hu CY, Ma LN, et al. Association between lipoprotein(a) levels and metabolic syndrome in a middle-aged and elderly Chinese cohort. Biomed Environ Sci. 2019;32:477-85.

42. Sung K, Wild SH, Byrne CD. Lipoprotein(a), metabolic syndrome and coronary calcium score in a large occupational cohort. Nutr Metab Cardiovasc Dis. 2013;23:1239-46.

43. Konerman M, Kulkarni K, Toth PP, Jones SR. Evidence of dependence of lipoprotein(a) on triglyceride and high-density lipoprotein metabolism. J Clin Lipidol. 2012;6:27-32.
44. Werba JP, Safa O, Gianfranceschi G, Michelagnoli S, Sirtori CR, Franceschini G. Plasma triglycerides and lipoprotein(a): inverse relationship in a hyperlipidemic Italian population. Atherosclerosis. 1993;101:203-11.

45. Bartens W, Rader DJ, Talley G, Brewer HB Jr. Decreased plasma levels of lipoprotein(a) in patients with hypertriglyceridemia. Atherosclerosis. 1994;108:147-9.

46. Argraves KMT, Kozarsky KF, Fallon JT, Harpel PC, Strickland DK. The atherogenic lipoprotein $\mathrm{Lp}(\mathrm{a})$ is internalized and degraded in a process mediated by the VLDL receptor. J Clin Invest. 1997;100:2170-81.

47. von Depka M, Nowak-Göttl U, Eisert R, Dieterich $C$, Barthels $M$, Scharrer I, et al. Increased lipoprotein (a) levels as an independent risk factor for venous thromboembolism. Blood. 2000;96:3364-8

48. Srikanthan K, Feyh A, Visweshwar H, Shapiro JI, Sodhi K. Systematic review of metabolic syndrome biomarkers: a panel for early detection, management, and risk stratification in the West Virginian population. Int J Med Sci. 2016;13:25-38.

49. Kostapanos MS, Florentin M, Elisaf MS, Mikhailidis DP. Hemostatic factors and the metabolic syndrome. Curr Vasc Pharmacol. 2013;11:880905.

50. Rangel-BaltazarE, Cuevas-Nasu L, Shamah-Levy T, Rodríguez-Ramírez S, Méndez-Gómez-Humarán I, Rivera JA. Association between high waistto-height ratio and cardiovascular risk among adults sampled by the 2016 half-way National Health and Nutrition Survey in Mexico (ENSANUT MC 2016). Nutrients. 2019;11:1402. 